\title{
Dynamic and Stability of Harmonic Driving Flexible Cartesian Robotic Arm with Bolted Joints Based on the Sensitivity and Multiple Scales Method
}

\author{
Yufei Liu, Wei Li, Xuefeng Yang, and Yuqiao Wang \\ School of Mechatronic Engineering, China University of Mining and Technology, Xuzhou 221116, China \\ Correspondence should be addressed to Xuefeng Yang; hopeasy@163.com
}

Received 29 September 2015; Accepted 22 November 2015

Academic Editor: Maria Gandarias

Copyright (C) 2015 Yufei Liu et al. This is an open access article distributed under the Creative Commons Attribution License, which permits unrestricted use, distribution, and reproduction in any medium, provided the original work is properly cited.

\begin{abstract}
Flexible Cartesian robotic arms (CRAs) are typical multicoupling systems. Considering the elastic effects of bolted joints and the motion disturbances, this paper investigates the dynamic and stability of the flexible CRA. With the kinetic energy and potential energy of the comprising components, Hamilton's variational principle and Duhamel integral are utilized to derive the dynamic equation and vibration differential equation. Based on the proposed elastic restraint model of the bolted joints, boundary conditions and mode equations of the flexible CRA are determined with using the principle of virtual work. According to the mode frequencies and sensitivities analysis, it reveals that the connecting stiffness of the bolted joints has significant influences, and the mode frequencies are more sensitive to the tensional stiffness. Moreover, describing the motion displacement of the driving base as combination of an average motion displacement and a harmonic disturbance, the vibration responses of the system are studied. The result indicates that the motion disturbance has obvious influence on the vibration responses, and the influence enhances under larger accelerating operations. The multiple scales method is introduced to analyze the parametric stability of the system, as well as the influences of the tensional stiffness and the end-effector on the stability.
\end{abstract}

\section{Introduction}

As one type of the typical industry robots, CRAs have been widely used in various industrial applications, such as injection-molding machines, loading and unloading robot in CNC lathes, welding robots, painting robots, and appendages attached to space vehicles [1-3]. As shown in Figure 1, the CRA can be modeled as a driving base, a manipulator, and an end-effector. Driven by the driving unit, for example, the motor, the manipulator can achieve 3-DOF translating motions and simultaneously the end-effector can execute the operating tasks, such as handing and selecting productions, welding, and painting. With the urgent requests of high productivity and high energy utilization efficiency, high operating speed and high operating accuracy are necessary for the robots. However, traditional rigid CRAs are constructed with heavy materials and bulky structure $[3,4]$, which are relatively heavy and limit the operating speed and contradict the purpose of improving the productivity and energy utilization efficiency. One of the significant measures to achieve the demand of lightweight and high operating speed is reducing the weight of main moving components, for example, the manipulators [3]. Different from the rigid CRAs, flexible CRAs use flexible manipulators which have light weights and can meet the purpose of high productivity and energy utilization efficiency and have been actively developed and received increasing attentions [3,5-9].

In the design stage, the connecting properties of the interface between the flexible manipulator and the driving base have significant effect on the dynamic characteristics of the system [10-12]. Generally, it is expected that the interface has sufficient connecting stiffness in the assembling process. However, it should be noted that, for the CRAs using bolted joints, the connecting stiffness is determined by the preloads; if the preloads are not controlled well in the assembling process, the connecting stiffness will reduce and be smaller than the expected value. Besides, with longtime executions especially accident collisions during the starting 


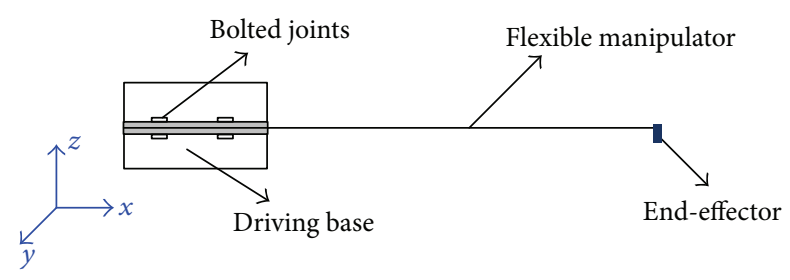

Figure 1: Schematic diagram of flexible Cartesian robotic arm with bolted joints.

and stopping, the bolted joints will loosen and the connecting stiffness reduces simultaneously. This insufficient or reduced connecting stiffness caused in the assembling process and executions all deviate the ideal status, and in this case the interface will exhibit certain elasticity. We can see that the flexible CRAs convey the coupling effects of rigid components and flexible components, which is a multibody system consisting of rigid body and multiflexible body. For this multibody system, the elastic deformations and residual vibrations will exhibit significant effect on the operating accuracy and service life especially under high speed tasks [3, 5], which are inevitable when executing the operation tasks, for example, changing its position by suddenly translating.

Besides, considering the coupling effects of the motor parameters and mechanism inertias, the motions of the driving base are not ideal constant and exhibit certain disturbances, especially for the system using harmonic drive in high speed operations [10]; the main reason for this is the unbalanced mass of motor in different rotational speeds and the backlash between the splines [13, 14]. Considering the motion disturbances, the system is similar to the parametrically excited system which has attracted numerous attentions especially in beams and plates investigations [15]. Describing the velocity as the combination of an average velocity and a disturbance, Chen et al. [16-18] intensively investigated the nonlinear vibrations and stabilities of axial moving beams. Yabuno and Nayfeh [19] investigated the nonlinear normal modes of a vertical cantilever beam excited by a principal parametric resonance. Feng and $\mathrm{Hu}[20]$ and $\mathrm{Li}$ and Zhang [21] studied the principal parametric and internal resonances of flexible beams and plates. For the driving base suffering harmonic excitations, Pratiher and Dwivedy [22-24] investigated the nonlinear vibrations of lateral moving CRAs, and in their investigations, however, the motion velocities or motion accelerations of the driving base are assumed as constant neglecting the influence of the motion disturbances; on the other hand, the restraint of the connecting interface is regarded as absolutely rigid and ignores the joint elasticity.

In this paper, we consider the rigid-flexible coupling effect and the motion disturbances caused by the effect of electromechanical couple; in this case, the flexible CRA studied here is a typical multicoupling system which is obviously different from the existent investigations. The sensitivity analysis and multiple scales method are introduced to investigate coupling dynamic and stability of the system, as well as the effect of the motion disturbances and the elastic

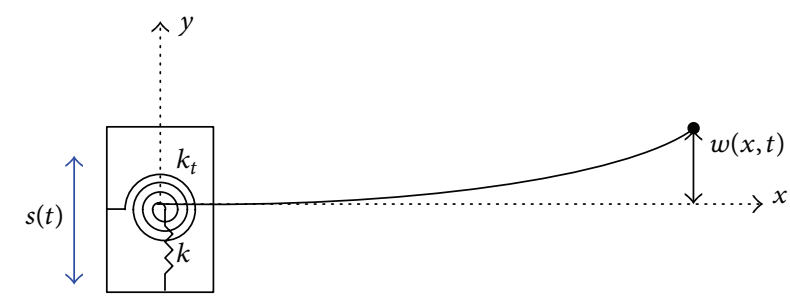

FIgURE 2: Elastic restraint model of the bolted joints.

restraint behavior of the bolted joints, which can direct the actual applications of the flexible CRA.

\section{Elastic Restraint Model}

For the CRA using bolted joints, it is expected that the interface between the flexible manipulator and the driving base has sufficient connecting stiffness in the assembling process. Generally, in the existent research, it is assumed that the interface is absolutely rigid and the restraint is ideal fixed. As detailed above, the connecting stiffness is accidently reduced and deviates the ideal status in the assembling process and during the executions. In this case, the interface is not absolutely rigid and exhibits certain elasticity, and the restraint is never ideally fixed. To accurately describe the connecting status of the bolted joints, the restraint of the interface is modeled as a tensional spring and a torsional spring which denote the tensional restraint effect and torsional restraint effect, respectively. The elastic restraint model of the bolted joints is proposed in Figure 2.

\section{Dynamic and Vibration Differential Equations}

Figure 2 further indicates that the flexible CRA system conveys rigid body and multiflexible body, and, for this multibody system, the behaviors of the flexible manipulator are coupled with the motions of the rigid driving base especially under high speed motions. To analyze the dynamic behaviors of the flexible manipulator, the motions of the rigid driving base should be considered. During the analysis, it is assumed that the flexible manipulator satisfies the Bernoulli-Euler Beam assumption and the transverse vibrations are the main considered motions.

The transverse vibration displacement of the flexible manipulator can be expressed as

$$
y(x, t)=s(t)+w(x, t),
$$

where $s(t)$ denotes the motion displacement of the driving base and $w(x, t)$ denotes the transverse vibration displacement of the flexible manipulator, and, according to the variables separation method [25], it yields

$$
w(x, t)=\sum_{i=1}^{n \rightarrow \infty} \phi_{i}(x) q_{i}(t),
$$

where $q(t)$ denotes the generalized coordinates and $\phi(x)$ denotes the mode shape of the flexible manipulator. 
The kinetic energy of the flexible CRA system can be written as

$$
\begin{aligned}
E_{k}= & \frac{1}{2} m_{s}\left(\frac{\mathrm{d} s}{\mathrm{~d} t}\right)^{2}+\frac{1}{2} m_{e}\left[\frac{\mathrm{d} s}{\mathrm{~d} t}+\frac{\partial w(L, t)}{\partial t}\right]^{2} \\
& +\frac{1}{2} \int_{0}^{L} \rho A\left(\frac{\mathrm{d} s}{\mathrm{~d} t}+\frac{\partial w}{\partial t}\right)^{2} \mathrm{~d} x,
\end{aligned}
$$

in which the first part denotes the kinetic energy of the driving base, the second part denotes the kinetic energy of the end-effector, the third part denotes the kinetic energy of the flexible manipulator, $m_{s}$ is the mass of the driving base, $m_{e}$ is the mass of the end-effector, and $\rho$ and $A$ are the mass density and cross-sectional area of the flexible manipulator, respectively.

The potential energy of the flexible CRA system mainly considers the elastic potential energy of the flexible manipulator and can be written as

$$
E_{p}=\frac{1}{2} \int_{0}^{L} E I\left(\frac{\partial^{2} w}{\partial x^{2}}\right)^{2} \mathrm{~d} x
$$

here $E$ is Young's modulus and $I$ is the cross-sectional moment of inertia about the neural axis and can be expressed as $I=b h^{3} / 12$, where $b$ and $h$ are the width and thickness of the flexible manipulator, respectively.

According to (2),

$$
\begin{aligned}
E_{k} & =\frac{1}{2} \sum_{i}\left[m_{e} \phi_{i}^{2}(L)+M_{i}\right]\left(\frac{\mathrm{d} q_{i}}{\mathrm{~d} t}\right)^{2} \\
& +\sum_{i}\left[m_{e} \phi_{i}(L)+\int_{0}^{L} \rho A \phi_{i} \mathrm{~d} x\right] \frac{\mathrm{d} s}{\mathrm{~d} t} \frac{\mathrm{d} q_{i}}{\mathrm{~d} t} \\
& +\frac{1}{2}\left(m_{s}+m_{e}+\rho A L\right)\left(\frac{\mathrm{d} s}{\mathrm{~d} t}\right)^{2} \\
E_{p} & =\frac{1}{2} \sum_{i} K_{i} q_{i}^{2},
\end{aligned}
$$

where $M_{i}$ and $K_{i}$ denote the generalized mass and the generalized stiffness of the $i$ th mode, respectively, which can be defined as

$$
\begin{aligned}
& M_{i}=\int_{0}^{L} \rho A \phi_{i}^{2} \mathrm{~d} x, \\
& K_{i}=\int_{0}^{L} E I\left(\frac{\mathrm{d}^{2} \phi_{i}}{\mathrm{~d} x^{2}}\right)^{2} \mathrm{~d} x .
\end{aligned}
$$

The Lagrangian function can be written as

$$
U=E_{k}-E_{p}
$$

According to Hamilton's variational principle [26], (7) satisfies following condition:

$$
\delta \int_{t_{0}}^{t_{1}} U \mathrm{~d} t=\int_{t_{0}}^{t_{1}} \delta\left(E_{k}-E_{p}\right) \mathrm{d} t=0
$$

where

$$
\begin{aligned}
\delta E_{k} & =\delta\left[\frac{1}{2} \sum_{i}\left[m_{e} \phi_{i}^{2}(L)+M_{i}\right]\left(\frac{\mathrm{d} q_{i}}{\mathrm{~d} t}\right)^{2}\right. \\
& +\sum_{i}\left[m_{e} \phi_{i}(L)+\int_{0}^{L} \rho A \phi_{i} \mathrm{~d} x\right] \frac{\mathrm{d} s}{\mathrm{~d} t} \frac{\mathrm{d} q_{i}}{\mathrm{~d} t} \\
& \left.+\frac{1}{2}\left(m_{s}+m_{e}+\rho A L\right)\left(\frac{\mathrm{d} s}{\mathrm{~d} t}\right)^{2}\right] \\
& =\sum_{i}\left[m_{e} \phi_{i}^{2}(L)+M_{i}\right] \frac{\mathrm{d} q_{i}}{\mathrm{~d} t} \delta \frac{\mathrm{d} q_{i}}{\mathrm{~d} t} \\
& +\left[m_{e} \phi_{i}(L)+\int_{0}^{L} \rho A \phi_{i} \mathrm{~d} x\right] \frac{\mathrm{d} s}{\mathrm{~d} t} \delta \frac{\mathrm{d} q_{i}}{\mathrm{~d} t} \\
& =\sum_{i}\left\{\left[m_{e} \phi_{i}^{2}(L)+M_{i}\right] \frac{\mathrm{d} q_{i}}{\mathrm{~d} t}\right. \\
& \left.+\left[m_{e} \phi_{i}(L)+\int_{0}^{L} \rho A \phi_{i} \mathrm{~d} x\right] \frac{\mathrm{d} s}{\mathrm{~d} t}\right\} \frac{\mathrm{d}}{\mathrm{d} t} \delta q_{i} \\
& =-\sum_{i}\left\{\left[m_{e} \phi_{i}^{2}(L)+M_{i}\right] \frac{\mathrm{d}^{2} q_{i}}{\mathrm{~d} t^{2}}\right. \\
& \left.+\left[m_{e} \phi_{i}(L)+\int_{0}^{L} \rho A \phi_{i} \mathrm{~d} x\right] \frac{\mathrm{d}^{2} s}{\mathrm{~d} t^{2}}\right\} \delta q_{i}, \\
\delta E_{p} & =\delta\left(\frac{1}{2} \sum_{i} K_{i} q_{i}^{2}\right)=\sum_{i} K_{i} q_{i} \delta q_{i} .
\end{aligned}
$$

Substituting (9) into (8), we obtain

$$
\begin{aligned}
& -\sum_{i}\left\{\left[m_{e} \phi_{i}^{2}(L)+M_{i}\right] \frac{\mathrm{d}^{2} q_{i}}{\mathrm{~d} t^{2}}\right. \\
& \left.+\left[m_{e} \phi_{i}(L)+\int_{0}^{L} \rho A \phi_{i} \mathrm{~d} x\right] \frac{\mathrm{d}^{2} s}{\mathrm{~d} t^{2}}+K_{i} q_{i}\right\} \delta q_{i} \\
& =0 .
\end{aligned}
$$

The dynamic equation can be further obtained as

$$
\begin{aligned}
& {\left[m_{e} \phi_{i}^{2}(L)+M_{i}\right] \ddot{q}_{i}+K_{i} q_{i}} \\
& \quad+\left[m_{e} \phi_{i}(L)+\int_{0}^{L} \rho A \phi_{i} \mathrm{~d} x\right] \ddot{s}=0,
\end{aligned}
$$

where $(\cdot)$ denotes the time derivative.

According to Duhamel integral [25], the vibration differential equation of the flexible manipulator can be written as

$$
\begin{aligned}
q_{i}(t)= & b_{1 i} \cos \omega_{i} t+b_{2 i} \sin \omega_{i} t \\
& +\frac{f_{i}}{K_{i}} \int_{0}^{t} \ddot{s}(\tau) \sin \omega_{i}(t-\tau) \mathrm{d} \tau,
\end{aligned}
$$


where

$$
\begin{aligned}
\omega_{i}^{2} & =\frac{K_{i}}{m_{e} \phi_{i}^{2}(L)+M_{i}}, \\
f_{i} & =-m_{e} \phi_{i}(L)-\int_{0}^{L} \rho A \phi_{i}(x) \mathrm{d} x, \\
b_{1 i} & =\frac{\rho A}{M_{i}} \int_{0}^{L} w(x, 0) \phi_{i} \mathrm{~d} x, \\
b_{2 i} & =\frac{\rho A}{M_{i} \omega_{i}} \int_{0}^{L} \frac{\partial w(x, 0)}{\partial t} \phi_{i} \mathrm{~d} x .
\end{aligned}
$$

As indicated in (11) and (12), the motion of the driving base $s(t)$ and the mode shape of the flexible manipulator $\phi(x)$ should be firstly determined for the dynamic equation and vibration differential equation.

\section{Mode and Sensitivity Analysis}

To determine the mode shapes $\phi(x)$, the principle of virtual work is utilized in this section. Defining the virtual displacement as $\delta w(x, t)$, according to the elastic restraint model in Figure 2, the fixed end and free end of the flexible manipulator satisfy

$$
\begin{aligned}
k w(0, t) \delta w+Q(0, t) \delta w & =0, \\
k_{t} \frac{\partial w(0, t)}{\partial x} \frac{\partial \delta w}{\partial x}+M(0, t) \frac{\partial \delta w}{\partial x} & =0, \\
Q(L, t) \delta w-m_{e} \frac{\partial^{2} w(L, t)}{\partial t^{2}} \delta w & =0, \\
M(L, t) & =0,
\end{aligned}
$$

where $Q(x, t)$ denotes the shear force of the flexible manipulator, $M(x, t)$ denotes the moment of the flexible manipulator, and, according to the small deflection assumption theory [27],

$$
\begin{aligned}
& Q(x, t)=E I \frac{\partial^{3} w(x, t)}{\partial x^{3}}, \\
& M(x, t)=E I \frac{\partial^{2} w(x, t)}{\partial x^{2}} .
\end{aligned}
$$

Combining (2), the boundary conditions of the flexible manipulator can be obtained from (14) as

$$
\begin{aligned}
k \phi(0)+E I \frac{\mathrm{d}^{3} \phi(0)}{\mathrm{d} x^{3}} & =0, \\
k_{t} \frac{\mathrm{d} \phi(0)}{\mathrm{d} x}+E I \frac{\mathrm{d}^{2} \phi(0)}{\mathrm{d} x^{2}} & =0, \\
\frac{\mathrm{d}^{3} \phi(L)}{\mathrm{d} x^{3}} & =-m_{e} \omega^{2} \phi(L), \\
\frac{\mathrm{d}^{2} \phi(L)}{\mathrm{d} x^{2}} & =0 .
\end{aligned}
$$

Describe the mode shape $\phi(x)$ as

$$
\begin{aligned}
\phi(x)= & \alpha_{1} \sin \beta x+\alpha_{2} \cos \beta x+\alpha_{3} \sinh \beta x \\
& +\alpha_{4} \cosh \beta x,
\end{aligned}
$$

where $\alpha_{1}, \alpha_{2}, \alpha_{3}, \alpha_{4}$ are coefficients determined by the boundary conditions and $\beta$ satisfies the relationship $\beta^{4}=$ $(\rho A / E I) \omega^{2}$.

Substituting (17) into (16) yields

$$
\left[\begin{array}{cc}
-\lambda \beta & k \\
k_{t} \beta & -\lambda
\end{array}\right]\left[\begin{array}{l}
\alpha_{1} \\
\alpha_{2}
\end{array}\right]+\left[\begin{array}{cc}
\lambda \beta & k \\
k_{t} \beta & \lambda
\end{array}\right]\left[\begin{array}{l}
\alpha_{3} \\
\alpha_{4}
\end{array}\right]=0,
$$

where $\lambda=E I \beta^{2}$, and

$$
\begin{aligned}
& {\left[\begin{array}{cc}
\psi \sin \beta L-\cos \beta L & \sin \beta L+\psi \cos \beta L \\
-\sin \beta L & -\cos \beta L
\end{array}\right]\left[\begin{array}{l}
\alpha_{1} \\
\alpha_{2}
\end{array}\right]} \\
& +\left[\begin{array}{cc}
\psi \sinh \beta L+\cosh \beta L & \sinh \beta L+\psi \cosh \beta L \\
\sinh \beta L & \cosh \beta L
\end{array}\right]\left[\begin{array}{l}
\alpha_{3} \\
\alpha_{4}
\end{array}\right] \\
& =0,
\end{aligned}
$$

where $\psi=(E I / \rho A) m_{e} \beta$.

$$
\text { After multiplying (18) with }
$$
$\left[\begin{array}{cc}\psi \sinh \beta L+\cosh \beta L & \sinh \beta L+\psi \cosh \beta L \\ \sinh \beta L & \cosh \beta L\end{array}\right]$, and before multiplying (19) with $\left[\begin{array}{ll}\lambda \beta & k \\ k_{t} \beta & \lambda\end{array}\right]$, through eliminating $\alpha_{3}$ and $\alpha_{4}$, and combining the coefficient of $\alpha_{1}$ and $\alpha_{2}$, we obtain

$$
\left[\begin{array}{ll}
\Delta_{11} & \Delta_{12} \\
\Delta_{21} & \Delta_{22}
\end{array}\right]\left[\begin{array}{l}
\alpha_{1} \\
\alpha_{2}
\end{array}\right]=0
$$

where

$$
\begin{aligned}
\Delta_{11}= & \lambda \beta(\cos \beta L-\cosh \beta L) \\
& +(k-\lambda \beta \psi)(\sin \beta L+\sinh \beta L), \\
\Delta_{12}= & -\lambda \beta(\sin \beta L+\sinh \beta L) \\
& +(k-\lambda \beta \psi)(\cos \beta L+\cosh \beta L), \\
\Delta_{21}= & k_{t} \beta(\cos \beta L+\cosh \beta L) \\
& +\left(\lambda-k_{t} \beta \psi\right)(\sin \beta L-\sinh \beta L), \\
\Delta_{22}= & -k_{t} \beta(\sin \beta L-\sinh \beta L) \\
& +\left(\lambda-k_{t} \beta \psi\right)(\cos \beta L-\cosh \beta L) .
\end{aligned}
$$

According to (20), the natural frequency equation can be obtained as

$$
\left|\begin{array}{ll}
\Delta_{11} & \Delta_{12} \\
\Delta_{21} & \Delta_{22}
\end{array}\right|=0
$$


which can be expressed as

$$
\begin{aligned}
& {\left[\left(k_{t} \lambda \beta^{2}+k \lambda\right)+\left(k_{t} \lambda \beta^{2} \psi-\lambda^{2} \beta-k k_{t} \beta\right) \psi\right] \cos \beta L} \\
& \quad \cdot \sinh \beta L \\
& \quad+\left[\left(k_{t} \lambda \beta^{2}-k \lambda\right)+\left(-k_{t} \lambda \beta^{2} \psi+\lambda^{2} \beta+k k_{t} \beta\right) \psi\right] \\
& \quad \cdot \sin \beta L \cosh \beta L-\left[\left(\lambda^{2}+k k_{t}\right) \beta-2 k_{t} \lambda \beta^{2} \psi\right] \\
& \cdot \cos \beta L \cosh \beta L+\left(\lambda^{2}-k k_{t}\right) \beta=0 .
\end{aligned}
$$

From (18), following relationship it can be obtained that

$$
\begin{aligned}
& \alpha_{3}=-\frac{k k_{t}+\lambda^{2}}{k k_{t}-\lambda^{2}} \alpha_{1}+\frac{2 k \lambda}{\left(k k_{t}-\lambda^{2}\right) \beta} \alpha_{2}, \\
& \alpha_{4}=\frac{2 k_{t} \lambda \beta}{k k_{t}-\lambda^{2}} \alpha_{1}-\frac{k k_{t}+\lambda^{2}}{k k_{t}-\lambda^{2}} \alpha_{2} .
\end{aligned}
$$

Substituting (24) into (17), the mode shape equation can be obtained as

$$
\begin{gathered}
\phi(x)=(\cos \beta x-\cosh \beta x)+\frac{2 k \lambda}{\left(k k_{t}-\lambda^{2}\right) \beta} \sinh \beta x \\
-\frac{2 \lambda^{2}}{k k_{t}-\lambda^{2}} \cosh \beta x+\zeta[(\sin \beta x-\sinh \beta x) \\
\left.-\frac{2 \lambda^{2}}{k k_{t}-\lambda^{2}} \sinh \beta x+\frac{2 k_{t} \lambda \beta}{k k_{t}-\lambda^{2}} \cosh \beta x\right],
\end{gathered}
$$

where

$$
\begin{aligned}
\zeta & =-\frac{\Delta_{12}}{\Delta_{11}} \\
& =\frac{-\lambda \beta(\sin \beta L+\sinh \beta L)+(k-\lambda \beta \psi)(\cos \beta L+\cosh \beta L)}{\lambda \beta(\cos \beta L-\cosh \beta L)+(k-\lambda \beta \psi)(\sin \beta L+\sinh \beta L)} .
\end{aligned}
$$

Assigning $k \rightarrow \infty$ and $k_{t} \rightarrow \infty,(23)$ and (25) become

$\psi \cos \omega L \sinh \omega L-\psi \sin \omega L \cosh \omega L$

$$
+\cos \omega L \cosh \omega L+1=0,
$$

$$
\begin{aligned}
& \phi(x)=\cos \beta x-\cosh \beta x \\
& -\frac{\cos \beta L+\cosh \beta L}{\sin \beta L+\sinh \beta L}(\sin \beta x-\sinh \beta x) .
\end{aligned}
$$

Neglecting the mass of the end-effector and assigning $m_{e}=0$ in (27) yield

$$
\cos \omega L \cosh \omega L+1=0 .
$$

Equations (28) and (29) are the mode shape equation and frequency equation of the flexible manipulator without the end-effector under the interface having absolutely fixed restraint which is consistent with the ideal rigid assumption in the existent literatures [25]. However, actually the assigned condition $k \rightarrow \infty$ and $k_{t} \rightarrow \infty$ is difficult to achieve; that is to say, the absolute fixed restraint only exists in the ideal situation and cannot reflect the actual restraint status while the proposed elastic restraint model can provide a whole restraint of the bolted joints.

To show the mode characteristic of the flexible CRA, the flexible manipulator is characterized by a flexible beam to describe its lower damp and stiffness properties with the structure parameters: $L=575 \mathrm{~mm}, b=28 \mathrm{~mm}, h=1 \mathrm{~mm}$, $E=197 \mathrm{GPa}, \rho=7850 \mathrm{~kg} / \mathrm{m}^{3}, \mu=0.26$, and $m_{e}=0.001 \mathrm{~kg}$.

By solving (23), the value of $\beta$ can be obtained, and, according to the relationship $\beta^{4}=(\rho A / E I) \omega^{2}$, the natural frequency of the flexible CRA can be expressed as

$$
\omega=\sqrt{\frac{E I}{\rho A}} \beta^{2} .
$$

Figures 3 and 4 show the influences of the tensional restraint stiffness $(k)$ and the torsional restraint stiffness $\left(k_{t}\right)$ on the mode frequencies. It should be noted that, for the ideal fixed restraint, the natural frequencies of the flexible manipulator can be calculated as $2.448 \mathrm{~Hz}, 15.339 \mathrm{~Hz}$, and $42.950 \mathrm{~Hz}$. As shown in Figure 3, with the decreasing of tensional restraint stiffness $(k)$, the first three natural frequencies obviously decrease and are small compared to the ideal fixed restraint. On the other hand, Figure 4 indicates that with torsional restraint stiffness $\left(k_{t}\right)$ decrease, the first natural frequency presents no change, while the second and third natural frequencies obviously increase. It can be seen that the connecting stiffness of the interface has significant effects on the mode characteristic, and the analysis results are different from the ideal status of fixed restraint. Moreover, the effect mechanisms of the tensional restraint stiffness and the torsional restraint stiffness are different.

To further detail the phenomenon shown in Figures 3 and 4 and further determine the main factor that should be significantly considered in the analysis, the sensitivity analysis is introduced.

According to (23), we define the following sensitivity function:

$$
\begin{aligned}
& S(\beta)=\left[\left(k_{t} \lambda \beta^{2}+k \lambda\right)+\left(k_{t} \lambda \beta^{2} \psi-\lambda^{2} \beta-k k_{t} \beta\right) \psi\right] \\
& \cdot \cos \beta L \sinh \beta L \\
& +\left[\left(k_{t} \lambda \beta^{2}-k \lambda\right)+\left(-k_{t} \lambda \beta^{2} \psi+\lambda^{2} \beta+k k_{t} \beta\right) \psi\right] \\
& \cdot \sin \beta L \cosh \beta L-\left[\left(\lambda^{2}+k k_{t}\right) \beta-2 k_{t} \lambda \beta^{2} \psi\right] \\
& \cdot \cos \beta L \cosh \beta L+\left(\lambda^{2}-k k_{t}\right) \beta .
\end{aligned}
$$

Based on (31), the sensitivities of the mode frequencies to the tensional restraint stiffness $(k)$ and the torsional restraint stiffness $\left(k_{t}\right)$ can be defined as

$$
\begin{aligned}
& \frac{\mathrm{d} \beta}{\mathrm{d} k}=\frac{\mathrm{d} \beta}{\mathrm{d} S} \cdot \frac{\mathrm{d} S}{\mathrm{~d} k}=\frac{\mathrm{d} S / \mathrm{d} k}{\mathrm{~d} S / \mathrm{d} \beta}, \\
& \frac{\mathrm{d} \beta}{\mathrm{d} k_{t}}=\frac{\mathrm{d} \beta}{\mathrm{d} S} \cdot \frac{\mathrm{d} S}{\mathrm{~d} k_{t}}=\frac{\mathrm{d} S / \mathrm{d} k_{t}}{\mathrm{~d} S / \mathrm{d} \beta} .
\end{aligned}
$$




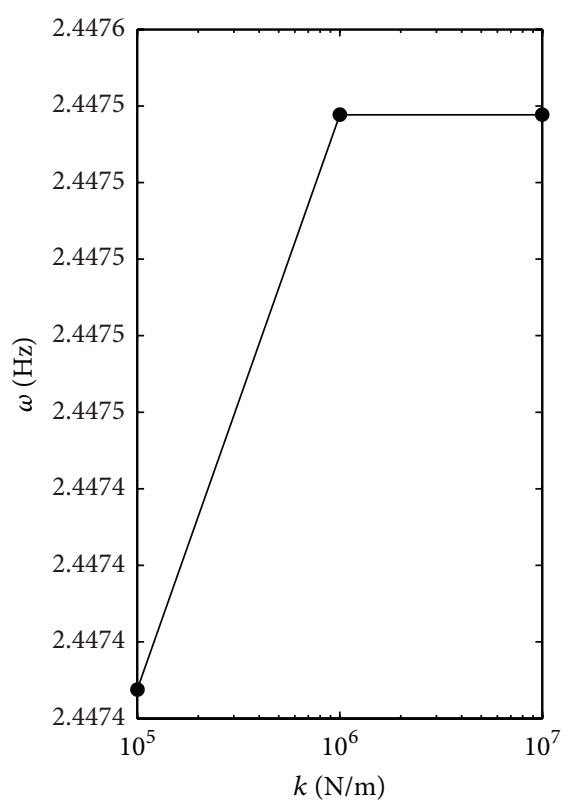

(a)

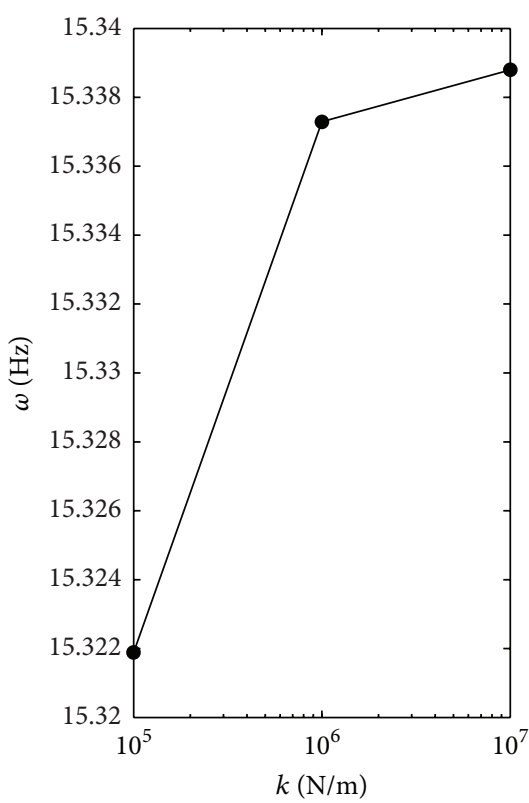

(b)

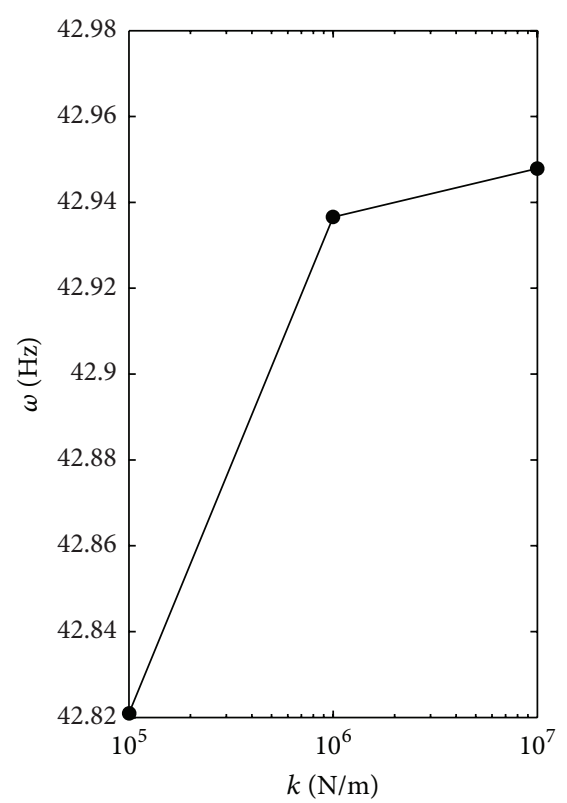

(c)

FIGURE 3: Influence of tensional restraint stiffness $(k)$ on the mode frequencies: (a) the first mode frequency; (b) the second mode frequency; (c) the third mode frequency.

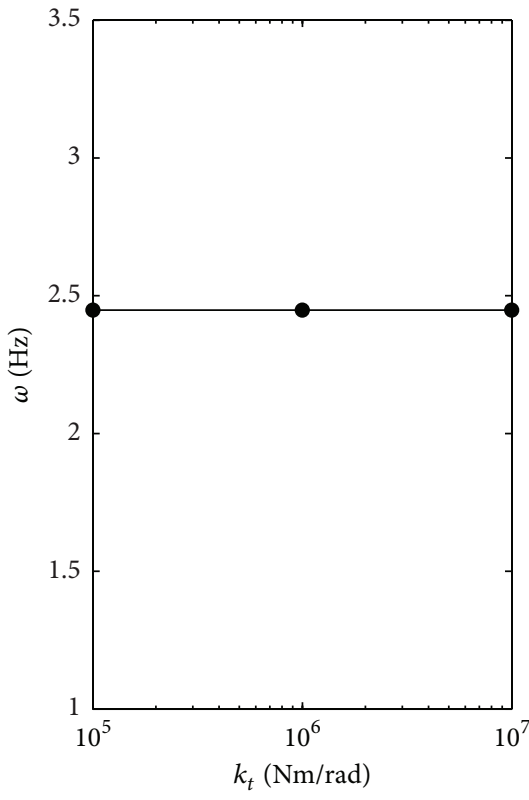

(a)

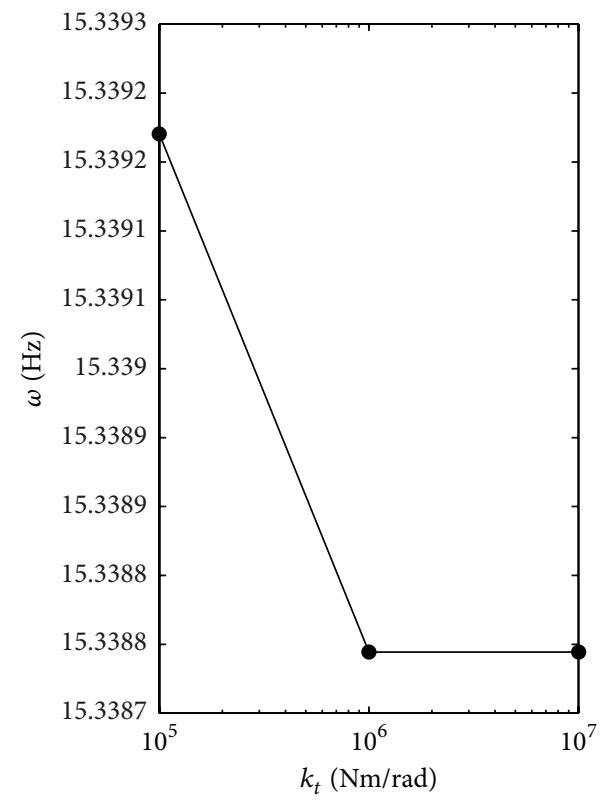

(b)

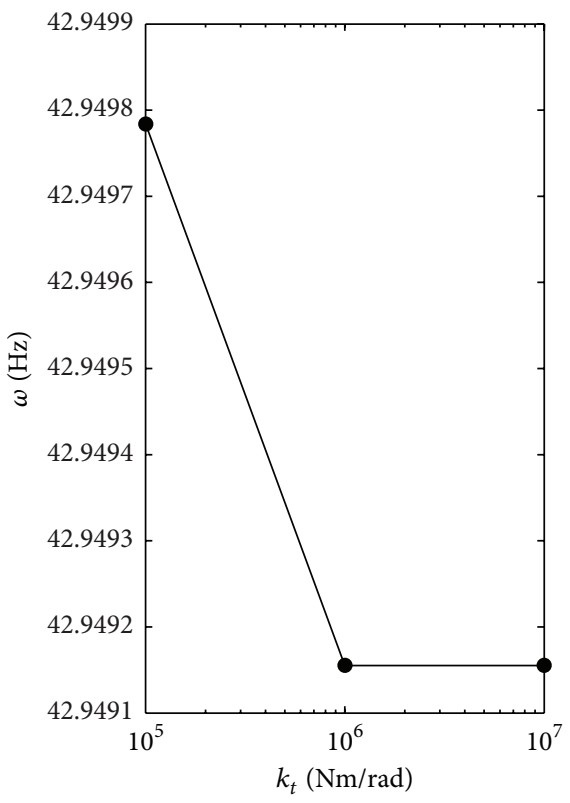

(c)

FIGURE 4: Influence of torsional restraint stiffness $\left(k_{t}\right)$ on the mode frequencies: (a) the first mode frequency; (b) the second mode frequency; (c) the third mode frequency.

Through the numerical method, we can obtain the sensitivities of the mode frequencies to the tensional restraint stiffness $(k)$ and the torsional restraint stiffness $\left(k_{t}\right)$, as shown in Figure 5. During the simulations, the tensional restraint stiffness is assigned as $k=1.0 \times 10^{5} \mathrm{~N} / \mathrm{m}, 1.0 \times 10^{6} \mathrm{~N} / \mathrm{m}$, and $1.0 \times 10^{7} \mathrm{~N} / \mathrm{m}$, and the torsional restraint stiffness is assigned as $k_{t}=1.0 \times 10^{5} \mathrm{Nm} / \mathrm{rad}, 1.0 \times 10^{6} \mathrm{Nm} / \mathrm{rad}$, and
$1.0 \times 10^{7} \mathrm{Nm} / \mathrm{rad}$. It can be seen that the sensitivity to $k$ is noticeable compared to $k_{t}$, and, with the decreasing of $k$, the sensitivity obviously enhances, while the sensitivity to $k_{t}$ presents tiny change. This indicates that, in this restraint status, the mode frequencies are more sensitive to the tensional stiffness, and the change of tensional stiffness has more obvious influence on the dynamic behaviors of the 


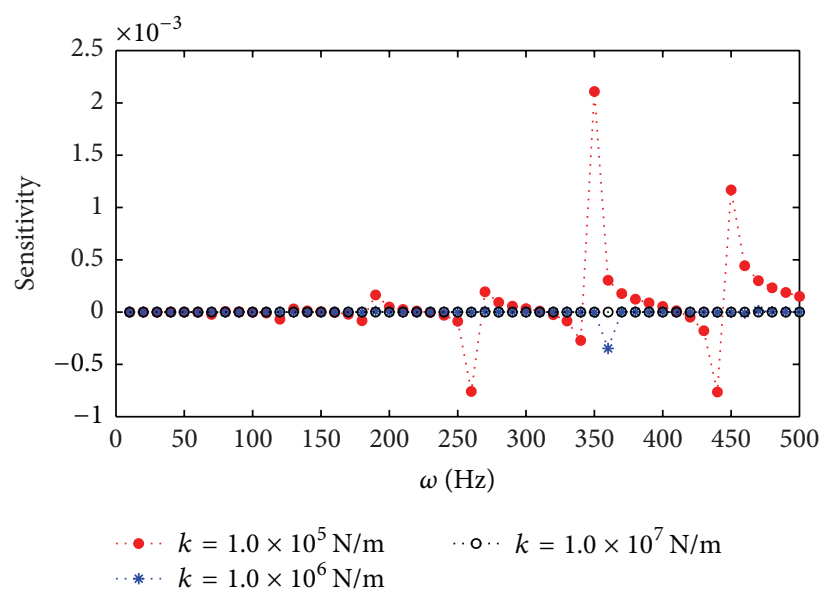

(a)

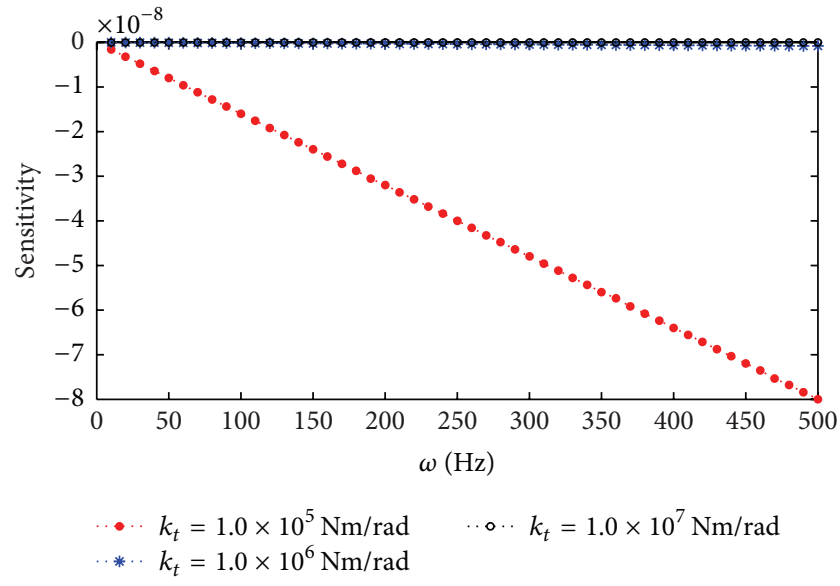

(b)

FIGURE 5: Sensitivities of the mode frequencies to the connecting stiffness: (a) sensitivity of the tensional restraint stiffness ( $k$ ); (b) sensitivity of the torsional restraint stiffness $\left(k_{t}\right)$.

system and more considerations in the design should be received.

\section{Vibration Responses and Stability}

5.1. Vibration Responses Characteristic. Investigating the vibration responses of the flexible manipulator, the motion of the driving base $s(t)$ should be determined. As detailed above, due to the electromechanical coupling effect, the driving motions of the driving base exhibit certain disturbances. From [28-30], we know that the motion disturbances have the features of harmonic functions, and, in [6], these harmonic motions are stated as the form of sinusoidal functions. Inspired by this, we expand the harmonic functions as the form of Fourier series and use sinusoidal functions to describe the disturbances. In this case, the motion of the driving base $s(t)$ can be written as

$$
s(t)=\frac{1}{2} a t^{2}+\gamma \sin \xi t
$$

where $a$ denotes the average motion acceleration of the driving base and $\gamma$ and $\xi$ denote the amplitude and frequency of the harmonic disturbance, respectively. In this case, (12) can be further expressed as

$$
\begin{aligned}
q_{i}(t)= & b_{1 i} \cos \omega_{i} t+b_{2 i} \sin \omega_{i} t \\
& +\frac{f_{i}}{K_{i}} \int_{0}^{t}\left(a-\gamma \xi^{2} \sin \xi \tau\right) \sin \omega_{i}(t-\tau) \mathrm{d} \tau \\
= & b_{1 i} \cos \omega_{i} t+b_{2 i} \sin \omega_{i} t-\frac{a f_{i}}{K_{i} \omega_{i}}\left(\cos \omega_{i} t-1\right) \\
& -\frac{\gamma f_{i}}{K_{i}} \frac{\xi \sin \omega_{i} t-\omega_{i} \sin \xi t}{1-\left(\omega_{i} / \xi\right)^{2}} .
\end{aligned}
$$

During the analysis, the frequency of the disturbance is assigned as $\xi=2 \omega_{1}$. Figure 6 shows the vibration responses of the flexible CRA system. We can see that the motion disturbance has obvious influence on the vibration responses. For the system with average velocity, the vibration response amplitude with motion disturbance $(\gamma=0.001 \mathrm{~mm})$ is smaller than the vibration response amplitude without motion disturbance $(\gamma=0 \mathrm{~mm})$; however, for the flexible CRA system with average acceleration, the vibration response amplitude with motion disturbance $(\gamma=0.001 \mathrm{~mm})$ is larger than the vibration response amplitude without motion disturbance $(\gamma=0 \mathrm{~mm})$, which indicates that the motion disturbance enhances the vibration of the end-effector especially for the accelerating operations.

According to the sensitivity analysis in Section 3, we have obtained that the mode frequency is more sensitive to the tensional stiffness $(k)$, which is the main factor that should be significantly considered in the dynamic analysis. Figure 7 shows the vibration responses of the end-effector under the motion disturbance. It can be seen that the vibration response amplitude of the end-effector with $k=1.0 \times 10^{5} \mathrm{~N} / \mathrm{m}$ is smaller than that of rigid restraint. Figure 8 shows the error of the vibration response amplitudes with and without the influence of the motion disturbance; it can be seen that the error of $k=1.0 \times 10^{5} \mathrm{~N} / \mathrm{m}$ is smaller than fixed restraint. This indicates that tensional stiffness has a suppressing effect on the motion disturbance.

The vibration responses of the end-effector under motion disturbance with different motion accelerations are shown in Figure 9. The accelerations are assigned as $a=0.1 \mathrm{~m} / \mathrm{s}^{2}$, $0.2 \mathrm{~m} / \mathrm{s}^{2}$, and $0.3 \mathrm{~m} / \mathrm{s}^{2}$. We can see that the vibration response amplitudes increase with the motion accelerations. Figure 10 further shows the error of the vibration response amplitudes with different motion accelerations; it can be seen that, with the increasing of the motion accelerations, the error aroused by the motion disturbance increases. This indicates that the influence of the motion disturbance will be enhanced under larger accelerating operations.

5.2. Parametric Stability Analysis Based on the Multiple Scales Method. From Figures 6-10, we can see that the motion 


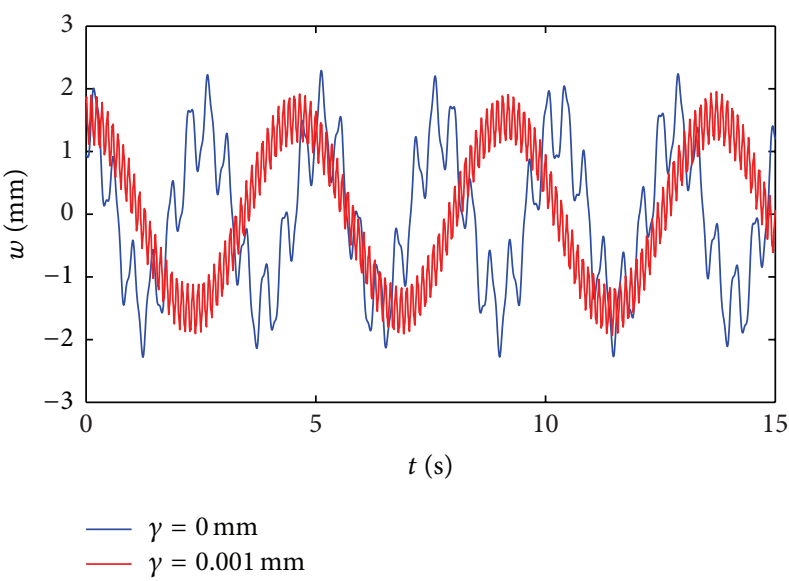

(a)

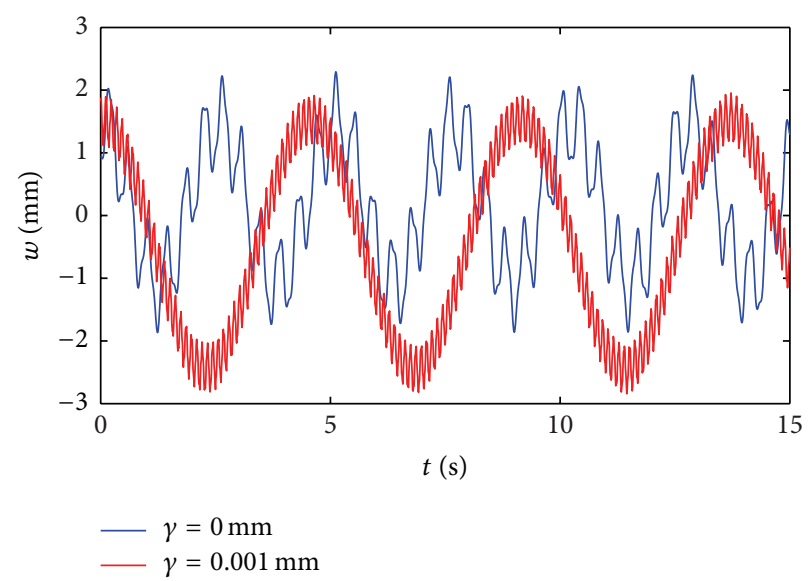

(b)

FIGURE 6: Vibration responses of the end-effector with and without the effect of the motion disturbance: (a) with average velocity; (b) with average acceleration.

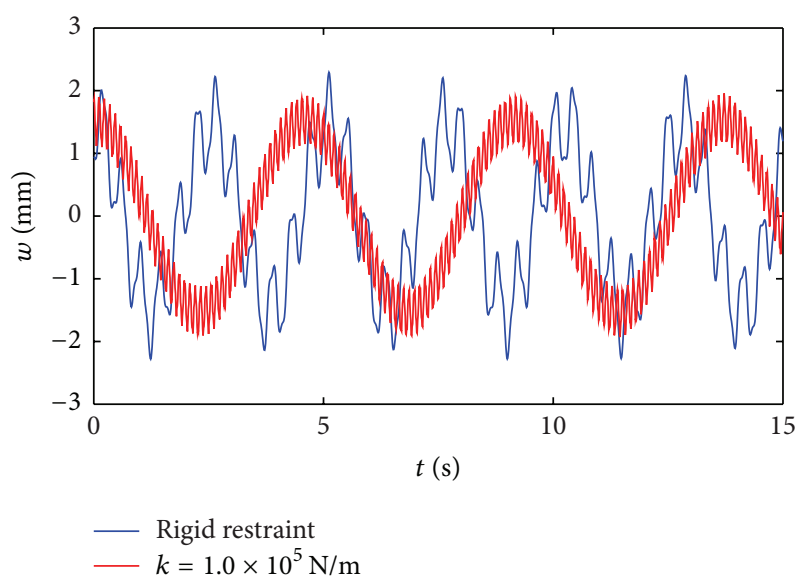

FIGURE 7: Vibration responses of the end-effector with rigid restraint and tensional stiffness $(k)$.

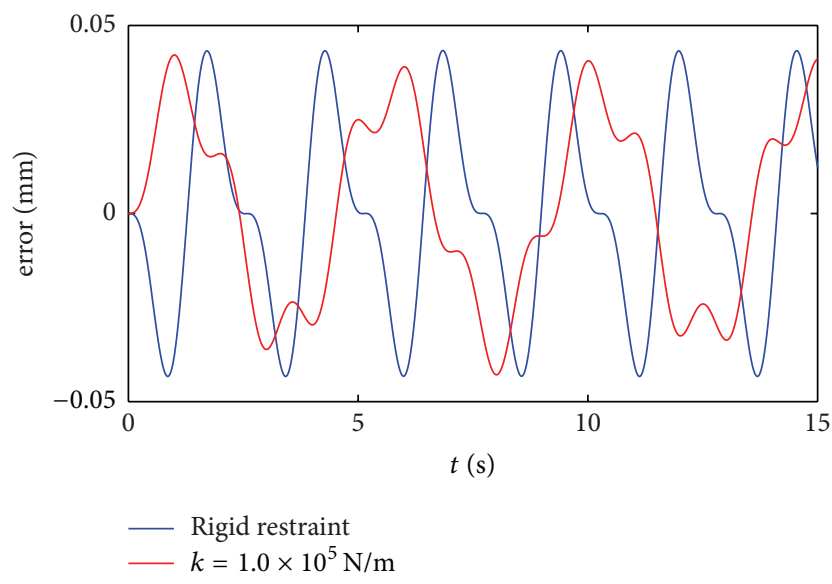

Figure 8: Effect of the tensional stiffness $(k)$ on the motion disturbance.

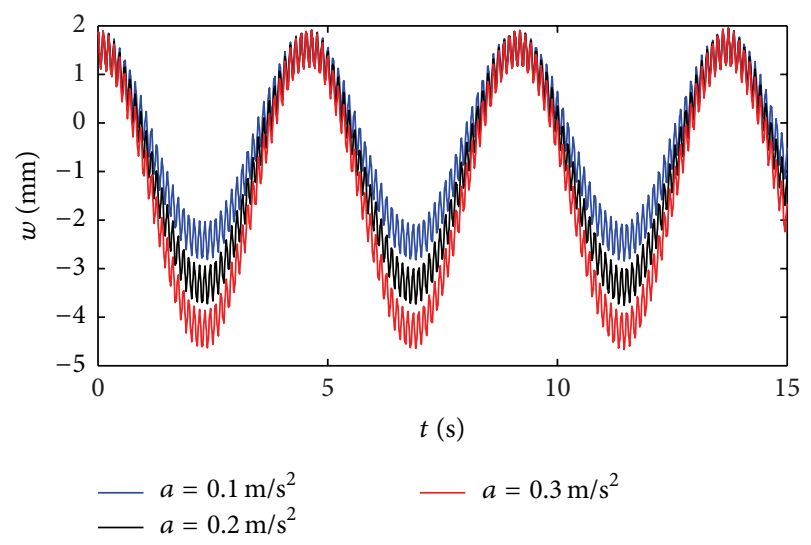

FIGURE 9: Vibration responses of the end-effector under different motion acceleration.

disturbance has obvious influence on the vibration responses of the system. In this section, we use the multiple scales method to analyze parametric stability.

Consider the displacement of the driving base to be a small simple harmonic variation about the constant mean displacement; namely,

$$
s(t)=\frac{1}{2} a t^{2}+\varepsilon \gamma \sin \xi t,
$$

where $\varepsilon$ stands for a small dimensionless parameter which is used to indicate the fact that the fluctuation amplitude is small.

Substituting (35) into (11) yields

$$
\begin{aligned}
& {\left[m_{e} \phi_{i}^{2}(L)+M_{i}\right] \ddot{q}_{i}+K_{i} q_{i}} \\
& \quad+\left[m_{e} \phi_{i}(L)+\int_{0}^{L} \rho A \phi_{i} \mathrm{~d} x\right]\left(a-\varepsilon \gamma \xi^{2} \sin \xi t\right) \\
& \quad=0 .
\end{aligned}
$$




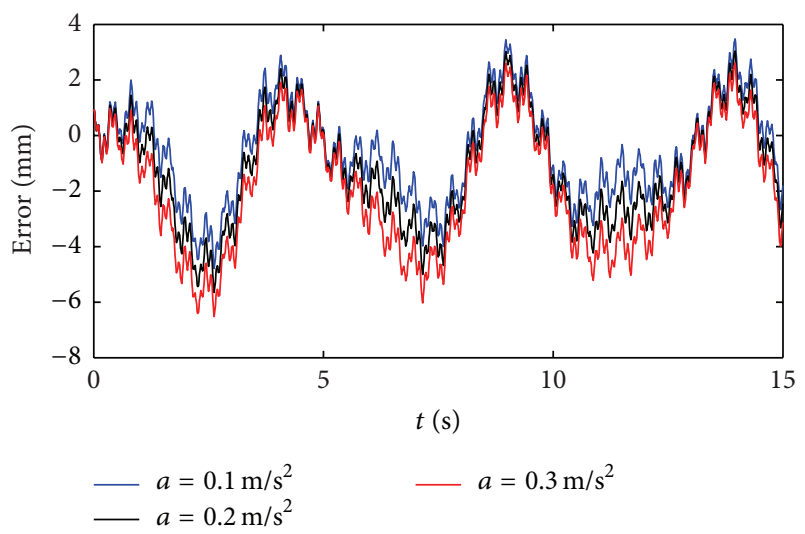

FIGURE 10: Effect of the motion accelerations on the motion disturbance.

Equation (36) defines a parametric vibration system, in which instability may occur under suitable conditions. The multiple scales method is employed to determine the stability boundary.

A first-order approximation solution of (36) is sought in the form

$$
q(t, \varepsilon)=q_{0}\left(T_{0}, T_{1}\right)+\varepsilon q_{0}\left(T_{0}, T_{1}\right)+O\left(\varepsilon^{2}\right),
$$

where $T_{0}=t$ is a fast scale characterizing motion and $T_{1}=$ $\varepsilon t$ is a slow scale characterizing motion and $O\left(\varepsilon^{2}\right)$ stands for higher order $\varepsilon^{2}$ terms.

Substituting (37) and following relationship

$$
\begin{gathered}
\frac{\partial}{\partial t}=\frac{\partial}{\partial T_{0}}+\varepsilon \frac{\partial}{\partial T_{1}}+O\left(\varepsilon^{2}\right) \\
\frac{\partial^{2}}{\partial t^{2}}=\frac{\partial^{2}}{\partial T_{0}^{2}}+2 \varepsilon \frac{\partial^{2}}{\partial T_{0} \partial T}+O\left(\varepsilon^{2}\right)
\end{gathered}
$$

into (36) and equalizing the coefficients of $\varepsilon^{0}$ and $\varepsilon^{1}$ in the resulting equation lead to

$$
\begin{aligned}
& {\left[m_{e} \phi_{i}^{2}(L)+M_{i}\right] \frac{\partial^{2} q_{0}}{\partial T_{0}{ }^{2}}+K_{i} q_{0}} \\
& +\left[m_{e} \phi_{i}(L)+\int_{0}^{L} \rho A \phi_{i} \mathrm{~d} x\right] a=0, \\
& {\left[m_{e} \phi_{i}^{2}(L)+M_{i}\right] \frac{\partial^{2} q_{1}}{\partial T_{0}{ }^{2}}} \\
& +K_{i} q_{1}=-2\left[m_{e} \phi_{i}^{2}(L)+M_{i}\right] \frac{\partial^{2} q_{0}}{\partial T_{0} \partial T_{1}} \\
& +\left[m_{e} \phi_{i}(L)+\int_{0}^{L} \rho A \phi_{i} \mathrm{~d} x\right] \gamma \xi^{2} \sin \xi t .
\end{aligned}
$$

According to the study of Wickert and Mote Jr. [31], the solution of (39) has the following form:

$$
q_{0}=A_{n}\left(T_{1}\right) e^{i \omega_{n} T_{0}}+\overline{A_{n}}\left(T_{1}\right) e^{-i \omega_{n} T_{0}},
$$

where the overbar denotes complex conjugation.
If the variation frequency $\xi$ approaches any natural frequency of the system, the parametric resonance may occur $[15,32]$. A detuning parameter $\sigma$ is introduced to quantify the deviation of $\xi$ from $\omega_{n}$, and in this case $\xi$ can be described as

$$
\xi=\omega_{n}+\varepsilon \sigma
$$

where $\omega_{n}$ is the $n$th natural frequency of system.

According to (41),

$$
\frac{\partial^{2} q_{0}}{\partial T_{0} \partial T_{1}}=i \omega_{n} \dot{A_{n}} e^{i \omega_{n} T_{0}}-i \omega_{n} \dot{A_{n}} e^{-i \omega_{n} T_{0}}
$$

Substituting (42) and (43) into (40) and expressing the trigonometric functions in exponential form yield

$$
\begin{aligned}
& {\left[m_{e} \phi_{i}^{2}(L)+M_{i}\right] \frac{\partial^{2} q_{1}}{\partial T_{0}^{2}}+K_{i} q_{1}=-2\left[m_{e} \phi_{i}^{2}(L)+M_{i}\right]} \\
& \cdot\left(i \omega_{n} \dot{A_{n}} e^{i \omega_{n} T_{0}}-i \omega_{n} \dot{A_{n}} e^{-i \omega_{n} T_{0}}\right)+\left[m_{e} \phi_{i}(L)\right. \\
& \left.+\int_{0}^{L} \rho A \phi_{i} \mathrm{~d} x\right] \gamma\left(\omega_{n}+\varepsilon \sigma\right)^{2} \\
& \quad+\frac{e^{i\left(\omega_{n}+\varepsilon \sigma\right) T_{0}}-e^{-i\left(\omega_{n}+\varepsilon \sigma\right) T_{0}}}{2 i}=-2 i \omega_{n}\left[m_{e} \phi_{i}^{2}(L)\right. \\
& \left.+M_{i}\right] \dot{A_{n}} e^{i \omega_{n} T_{0}}-\frac{1}{2} i\left[m_{e} \phi_{i}(L)+\int_{0}^{L} \rho A \phi_{i} \mathrm{~d} x\right] \\
& +\gamma\left(\omega_{n}^{2}+2 \omega_{n} \varepsilon \sigma+\varepsilon^{2} \sigma^{2}\right) e^{i \sigma T_{1}} \cdot e^{i \omega_{n} T_{0}}+\mathrm{cc} \\
& \quad=-2 i \omega_{n}\left\{\left[m_{e} \phi_{i}^{2}(L)+M_{i}\right] \dot{A_{n}}\right. \\
& \left.+\frac{1}{4} \gamma \omega_{n}\left[m_{e} \phi_{i}(L)+\int_{0}^{L} \rho A \phi_{i} \mathrm{~d} x\right] e^{i \sigma T_{1}}\right\} e^{i \omega_{n} T_{0}}+\mathrm{cc} \\
& +\mathrm{NST},
\end{aligned}
$$

where the dot denotes the derivation with respect to the slow time variable $T_{1}$, cc denotes the complex conjugate of all preceding terms on the right hand, and NST stands for the terms that will not bring secular terms into the solution.

Equation (44) has a bounded solution only if a solvability condition holds [33]. The solvability condition demands the orthogonal relationships

$$
\begin{aligned}
& \left\langle\left[m_{e} \phi_{i}^{2}(L)+M_{i}\right] \dot{A_{n}}\right. \\
& \left.\quad+\frac{1}{4} \gamma \omega_{n}\left[m_{e} \phi_{i}(L)+\int_{0}^{L} \rho A \phi_{i} \mathrm{~d} x\right] e^{i \sigma T_{1}}, \phi_{n}\right\rangle=0,
\end{aligned}
$$

where the inner product is defined for complex functions on $[0,1]$ as

$$
\langle f, g\rangle=\int_{0}^{1} f \bar{g} \mathrm{~d} x
$$



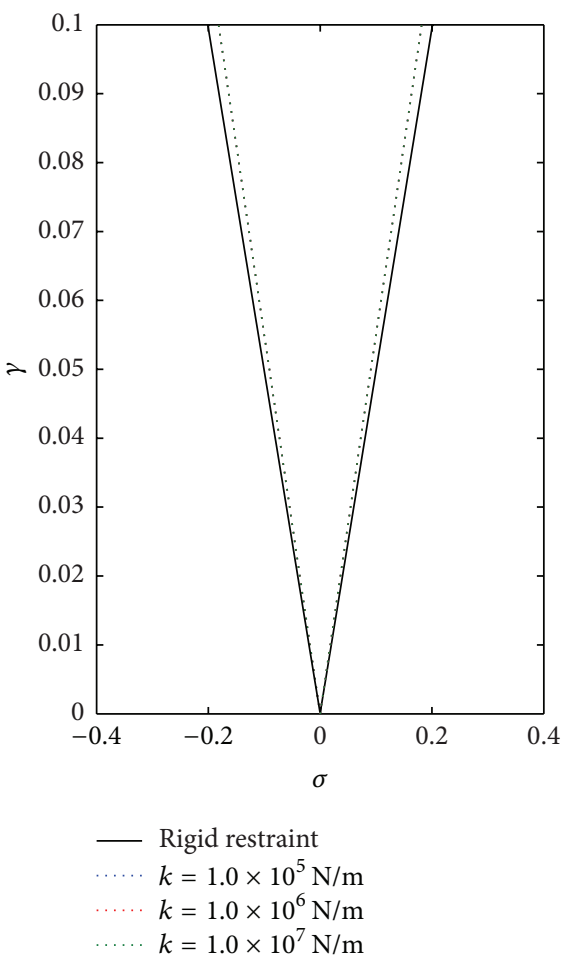

(a)

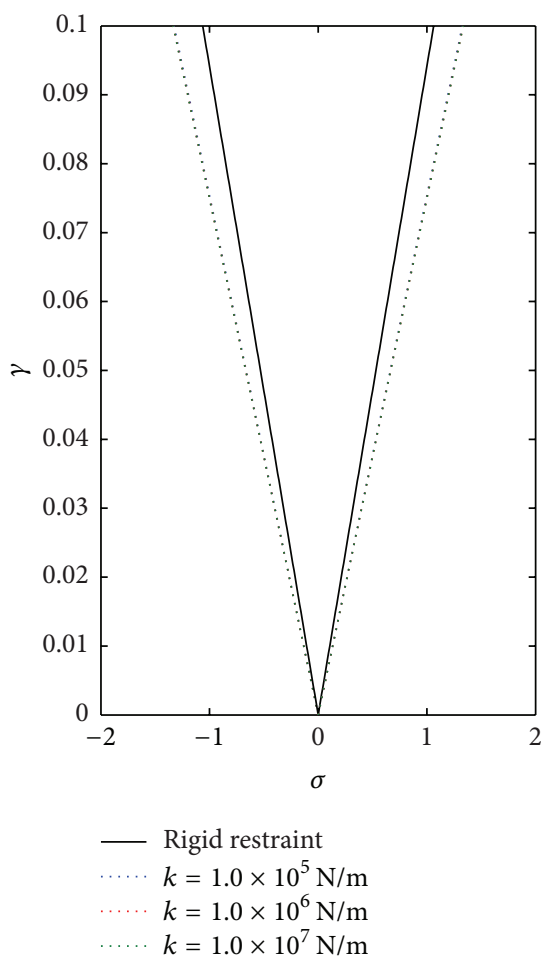

(b)

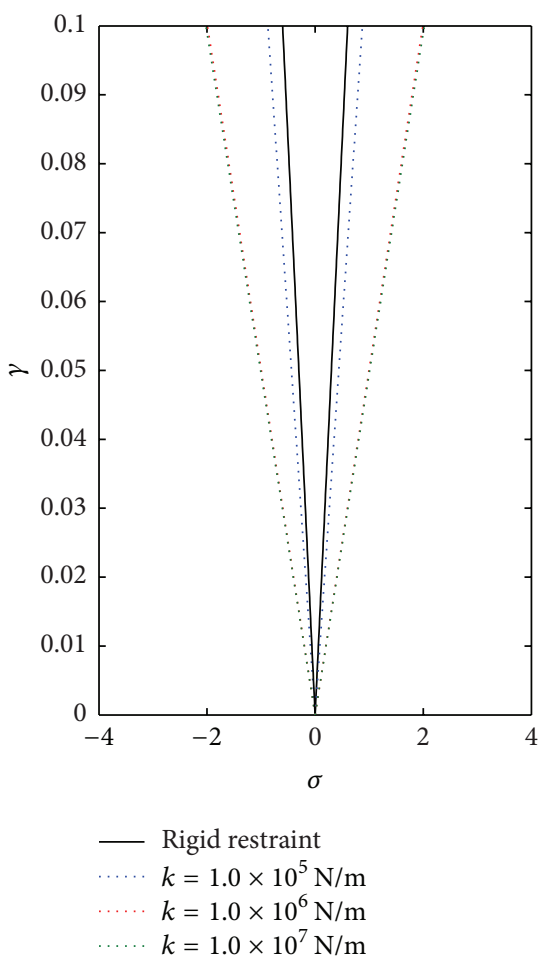

(c)

FIGURE 11: Influence of the tensional stiffness on the stability boundaries for the first three principal resonances: (a) the first principal resonance; (b) the second principal resonance; (c) the third principal resonance.

Application of the distributive law of the inner product to (45) leads to

$$
\begin{aligned}
& {\left[m_{e} \phi_{i}^{2}(L)+M_{i}\right] \dot{A_{n}}} \\
& \quad+\frac{1}{4} \gamma \omega_{n}\left[m_{e} \phi_{i}(L)+\int_{0}^{L} \rho A \phi_{i} \mathrm{~d} x\right] e^{i \sigma T_{1}} \int_{0}^{1} \overline{\phi_{n}} d x \\
& \quad=0
\end{aligned}
$$

namely,

$$
\begin{aligned}
& {\left[m_{e} \phi_{i}^{2}(L)+M_{i}\right] \dot{A_{n}}} \\
& \quad+\frac{1}{4} \gamma \omega_{n}\left[m_{e} \phi_{i}(L)+\int_{0}^{L} \rho A \phi_{i} \mathrm{~d} x\right] e^{i \sigma T_{1}}=0 .
\end{aligned}
$$

Equation (48) keeps similar form to the resulting equation in $[16,17]$; thus, according to $[16,17]$, the stability boundary can be similarly expressed by

$$
\sigma= \pm \frac{1}{4} \frac{m_{e} \phi_{i}(L)+\int_{0}^{L} \rho A \phi_{i} \mathrm{~d} x}{m_{e} \phi_{i}^{2}(L)+M_{i}}\left|\omega_{n}\right| \gamma
$$

The stability boundaries of the system for the first three principal resonances in plane $\sigma-\gamma$ are shown in Figure 11. The tensional stiffness is assigned as $k=1.0 \times 10^{5} \mathrm{~N} / \mathrm{m}$, $1.0 \times 10^{6} \mathrm{~N} / \mathrm{m}$, and $1.0 \times 10^{7} \mathrm{~N} / \mathrm{m}$. It can be seen that, with the change of the tensional stiffness, the instability zone of the first principal resonance presents minor change while the instability regions of the second and third principal resonances become wider. This indicates that the connecting stiffness has influence on the stability of the system.

Figure 12 presents the stability boundaries of the system with different end-effector. The mass of the end-effector is assigned as $m_{e}=0 \mathrm{~kg}, 0.001 \mathrm{~kg}$, and $0.005 \mathrm{~kg}$. It can be seen that with the increasing of the mass, the instability regions of the first three principal resonances become narrower, which indicates that a suitable lager mass end-effector has effect on the stability of the system and this is meaningful for the structure design of the flexible CRA system.

\section{Conclusions}

Considering the effects of rigid-flexible couples and parametric excitations, the flexible CRA system investigated remains a multicoupling system, and this paper studied the dynamic and stability of this system. It demonstrated that, considering the elastic restraint behavior of the bolted joints, the dynamic characteristics are different from the ideal fixed restraint in the absolutely rigid assumption. The connecting stiffness of the bolted joints has significant effect on the mode characteristic and the mode frequencies are more sensitive to the tensional stiffness. Moreover, the motion disturbance has obvious influence on the vibration responses, especially for the system having accelerating motions, and the vibration response amplitudes become larger which indicates that the motion disturbance enhances the vibration of the 


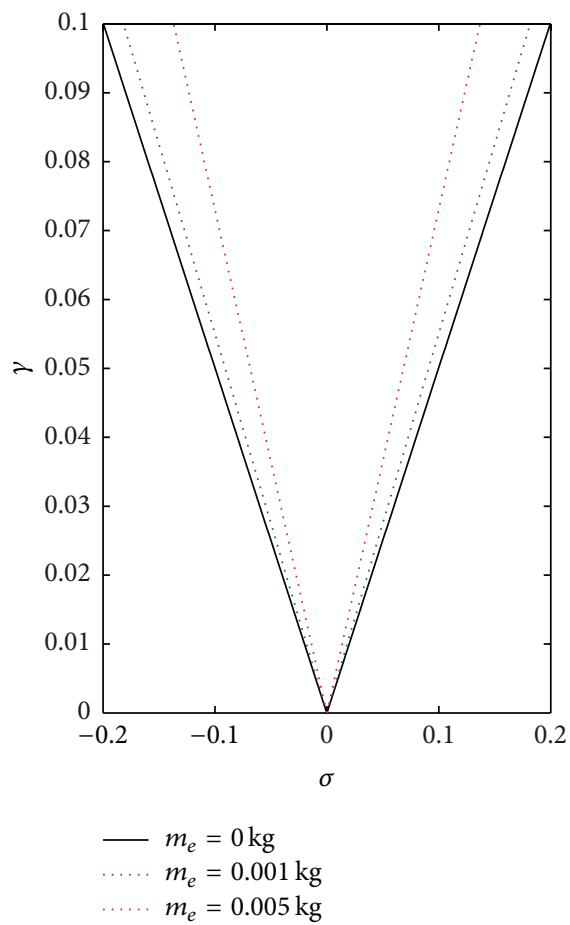

(a)

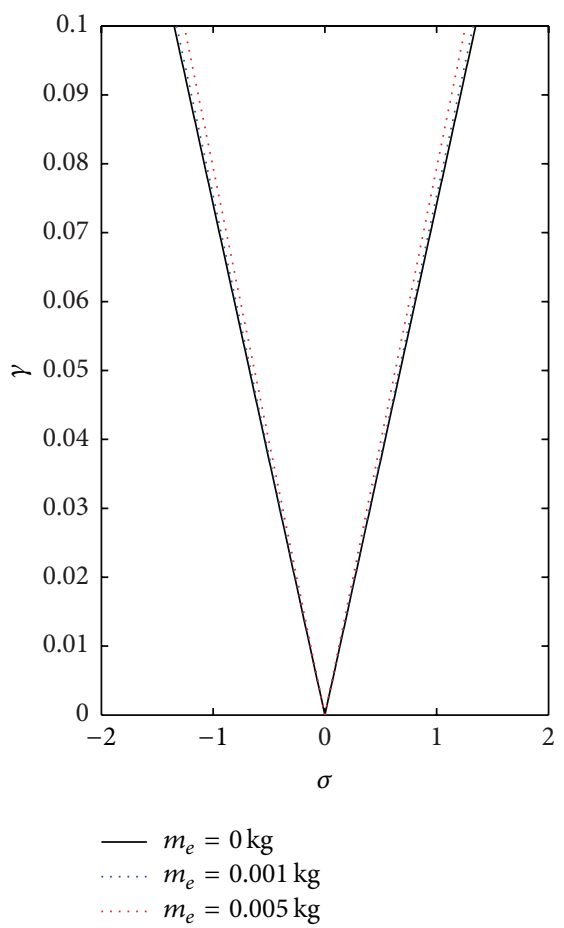

(b)

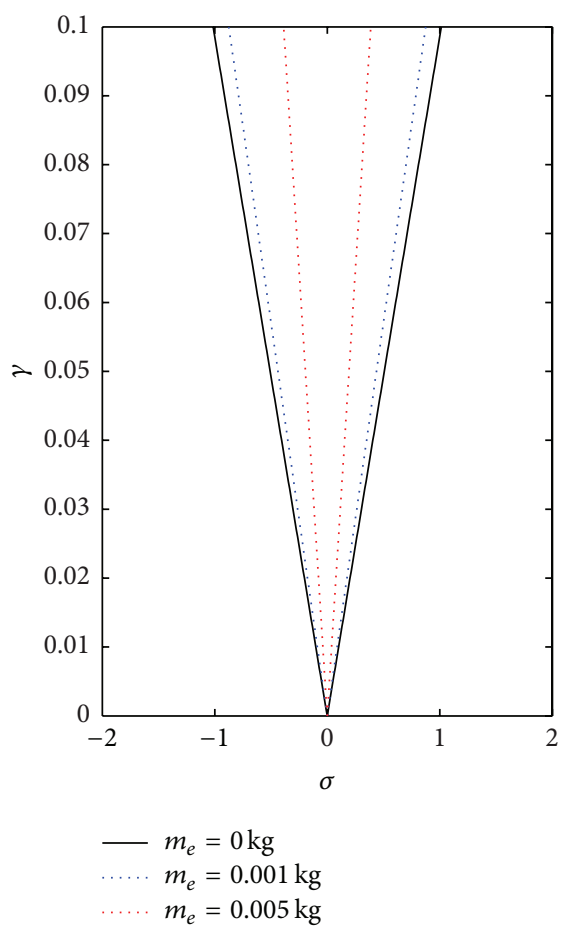

(c)

FIGURE 12: Influence of the end-effector on the stability boundaries for the first three principal resonances: (a) the first principal resonance; (b) the second principal resonance; (c) the third principal resonance.

end-effector; moreover, the influence will be enhanced under larger accelerating operations. The multiple scales method is introduced to analyze the parametric stability and obtain the stability boundary of the system; the result reveals that the tensional stiffness has obvious influence on the stability; with the change of the tensional stiffness, the instability region of the first principal resonance presents minor change while the instability regions of the second and third principal resonances become wider; with the increasing of the mass of the end-effector, the instability zones of the first three principal resonances become narrower, which indicates that a suitable lager mass end-effector has effect on the stability of the system. This study is meaningful for the structure parameters design, motions optimization, and further vibration control of robotic arms.

\section{Conflict of Interests}

The authors declare that there is no conflict of interests regarding the publication of this paper.

\section{Acknowledgments}

This research work is supported by the National Natural Science Foundation of China (no. 51305444), the Doctoral Fund of Ministry of Education (no. 20120095120013), the Scientific and Technological Project of Jiangsu Province (BY201402806), and the Project Funded by the Priority Academic Program Development of Jiangsu Higher Education Institutions (PAPD). The authors sincerely thank the reviewers for their constructive comments which are substantially helpful for improving the paper and our further work.

\section{References}

[1] B. Kim and J. Chung, "Residual vibration reduction of a flexible beam deploying from a translating hub," Journal of Sound and Vibration, vol. 333, no. 16, pp. 3759-3775, 2014.

[2] C. H. Park, D. I. Park, and J. H. Park, "Vibration control of flexible mode for a beam-type substrate transport robot," International Journal of Advanced Robotic Systems, vol. 10, pp. 1-7, 2013.

[3] S. K. Dwivedy and P. Eberhard, "Dynamic analysis of flexible manipulators, a literature review," Mechanism and Machine Theory, vol. 41, no. 7, pp. 749-777, 2006.

[4] M. H. Shaheed, H. Poerwanto, and M. O. Tokhi, "Adaptive inverse-dynamic and neuro-inverse-dynamic active vibration control of a single-link flexible manipulator," Proceedings of the Institution of Mechanical Engineers I: Journal of Systems and Control Engineering, vol. 219, no. 6, pp. 431-448, 2005.

[5] H. N. Rahimi and M. Nazemizadeh, "Dynamic analysis and intelligent control techniques for flexible manipulators: a review," Advanced Robotics, vol. 28, no. 2, pp. 63-76, 2014.

[6] J. L. Zhao, S. Z. Yan, and J. N. Wu, "Analysis of parameter sensitivity of space manipulator with harmonic drive based on the revised response surface method," Acta Astronautica, vol. 98, no. 1, pp. 86-96, 2014.

[7] S. Seung, P. Liu, S. Park, J.-O. Park, and S. Y. Ko, "Single-port robotic manipulator system for brain tumor removal surgery: SiromanS," Mechatronics, vol. 26, pp. 16-28, 2015. 
[8] S. E. Butner and M. Ghodoussi, "Transforming a surgical robot for human telesurgery," IEEE Transactions on Robotics and Automation, vol. 19, no. 5, pp. 818-824, 2003.

[9] Y. M. Li, H. Tang, Q. S. Xu, and Y. Yun, "Development status of micromanipulator technology for biomedical applications," Chinese Journal of Mechanical Engineering, vol. 47, no. 23, pp. 1-13, 2011.

[10] B. Siciliano and O. Khatib, Handbook of Robotics, Springer, London, UK, 2008.

[11] T. N. Guo, L. Li, L. G. Cai, and Y. S. Zhao, "Alternative method for identification of the dynamic properties of bolted joints," Journal of Mechanical Science and Technology, vol. 26, no. 10, pp. 3017-3027, 2012.

[12] J. H. Wang and S. C. Chuang, "Reducing errors in the identification of structural joint parameters using error functions," Journal of Sound and Vibration, vol. 273, no. 1-2, pp. 295-316, 2004.

[13] C. Lee and C. Chen, "Experimental application of a vibration absorber in structural vibration reduction using tunable fluid mass driven by micropump," Journal of Sound and Vibration, vol. 348, pp. 31-40, 2015.

[14] T. D. Tuttle and W. P. Seering, "A nonlinear model of a harmonic drive gear transmission," IEEE Transactions on Robotics and Automation, vol. 12, no. 3, pp. 368-374, 1996.

[15] I. F. Tho and N. Henk, Parametric Resonance in Dynamical Systems, Springer, New York, NY, USA, 2012.

[16] L.-Q. Chen and X.-D. Yang, "Stability in parametric resonance of axially moving viscoelastic beams with time-dependent speed," Journal of Sound and Vibration, vol. 284, no. 3-5, pp. 879-891, 2005.

[17] X.-D. Yang and L.-Q. Chen, "Stability in parametric resonance of axially accelerating beams constituted by Boltzmann's superposition principle," Journal of Sound and Vibration, vol. 289, no. 1-2, pp. 54-65, 2006.

[18] H. Ding and L. Q. Chen, "Nonlinear dynamics of axially accelerating viscoelastic beams based on differential quadrature," Acta Mechanica Solida Sinica, vol. 22, no. 3, pp. 267-275, 2009.

[19] H. Yabuno and A. H. Nayfeh, "Nonlinear normal modes of a parametrically excited cantilever beam," Nonlinear Dynamics, vol. 25, no. 1-3, pp. 65-77, 2001.

[20] Z. H. Feng and H. Y. Hu, "Principal parametric and three-to-one internal resonances of flexible beams undergoing a large linear motion," Acta Mechanica Sinica, vol. 19, no. 4, pp. 355-364, 2003.

[21] S.-B. Li and W. Zhang, "Global bifurcations and multi-pulse chaotic dynamics of rectangular thin plate with one-to-one internal resonance," Applied Mathematics and Mechanics, vol. 33, no. 9, pp. 1115-1128, 2012.

[22] B. Pratiher, "Non-linear response of a magneto-elastic translating beam with prismatic joint for higher resonance conditions," International Journal of Non-Linear Mechanics, vol. 46, no. 5, pp. 685-692, 2011.

[23] B. Pratiher and S. K. Dwivedy, "Nonlinear response of a flexible Cartesian manipulator with payload and pulsating axial force," Nonlinear Dynamics, vol. 57, no. 1-2, pp. 177-195, 2009.

[24] B. Pratiher and S. K. Dwivedy, "Non-linear dynamics of a flexible single link Cartesian manipulator," International Journal of Non-Linear Mechanics, vol. 42, no. 9, pp. 1062-1073, 2007.

[25] S. R. Singiresu, Mechanical Vibration, Pearson Education, 4th edition, 2004.

[26] M. Fengxiang, Analytical Mechanics, Beijing Institute of Technology Press, Beijing, China, 2013.
[27] D. Gross, W. Hauger, J. Schröder, W. A. Wall, and J. Bonet, Engineering Mechanics 2: Mechanics of Materials, Springer, London, UK, 2011.

[28] F. W. Liou, A. G. Erdman, and C. S. Lin, "Dynamic analysis of a motor-gear-mechanism system," Mechanism and Machine Theory, vol. 26, no. 3, pp. 239-252, 1991.

[29] H. S. Yan, M. C. Tsai, and M. H. Hsu, "An experimental study of the effects of cam speeds on cam-follower systems," Mechanism and Machine Theory, vol. 31, no. 4, pp. 397-412, 1996.

[30] L. Cveticanin, "Stability of motion of the cam-follower system," Mechanism and Machine Theory, vol. 42, no. 9, pp. 1238-1250, 2007.

[31] J. A. Wickert and C. D. Mote Jr., "Classical vibration analysis of axially moving continua," Journal of Applied Mechanics, vol. 57, no. 3, pp. 738-744, 1990.

[32] A. H. Nayfeh and D. T. Mook, Nonlinear Oscillations, John Wiley \& Sons, Ontario, Canada, 1995.

[33] L.-Q. Chen and J. W. Zu, "Solvability condition in multi-scale analysis of gyroscopic continua," Journal of Sound and Vibration, vol. 309, no. 1-2, pp. 338-342, 2008. 


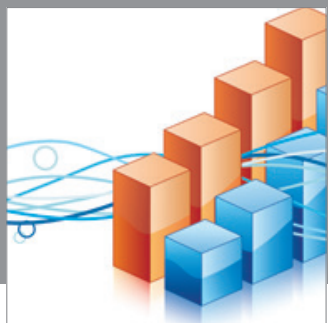

Advances in

Operations Research

mansans

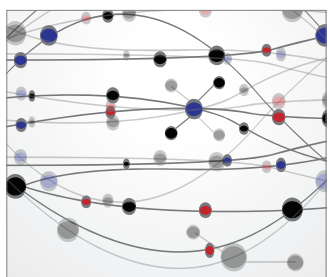

The Scientific World Journal
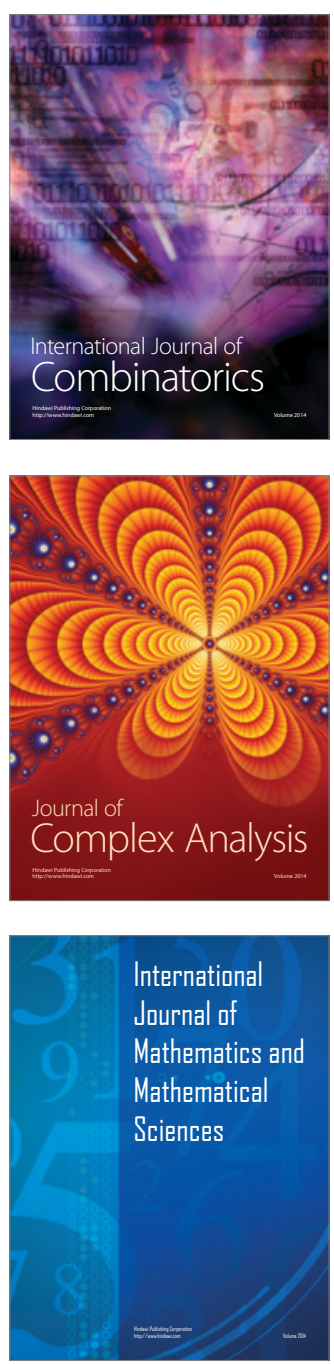
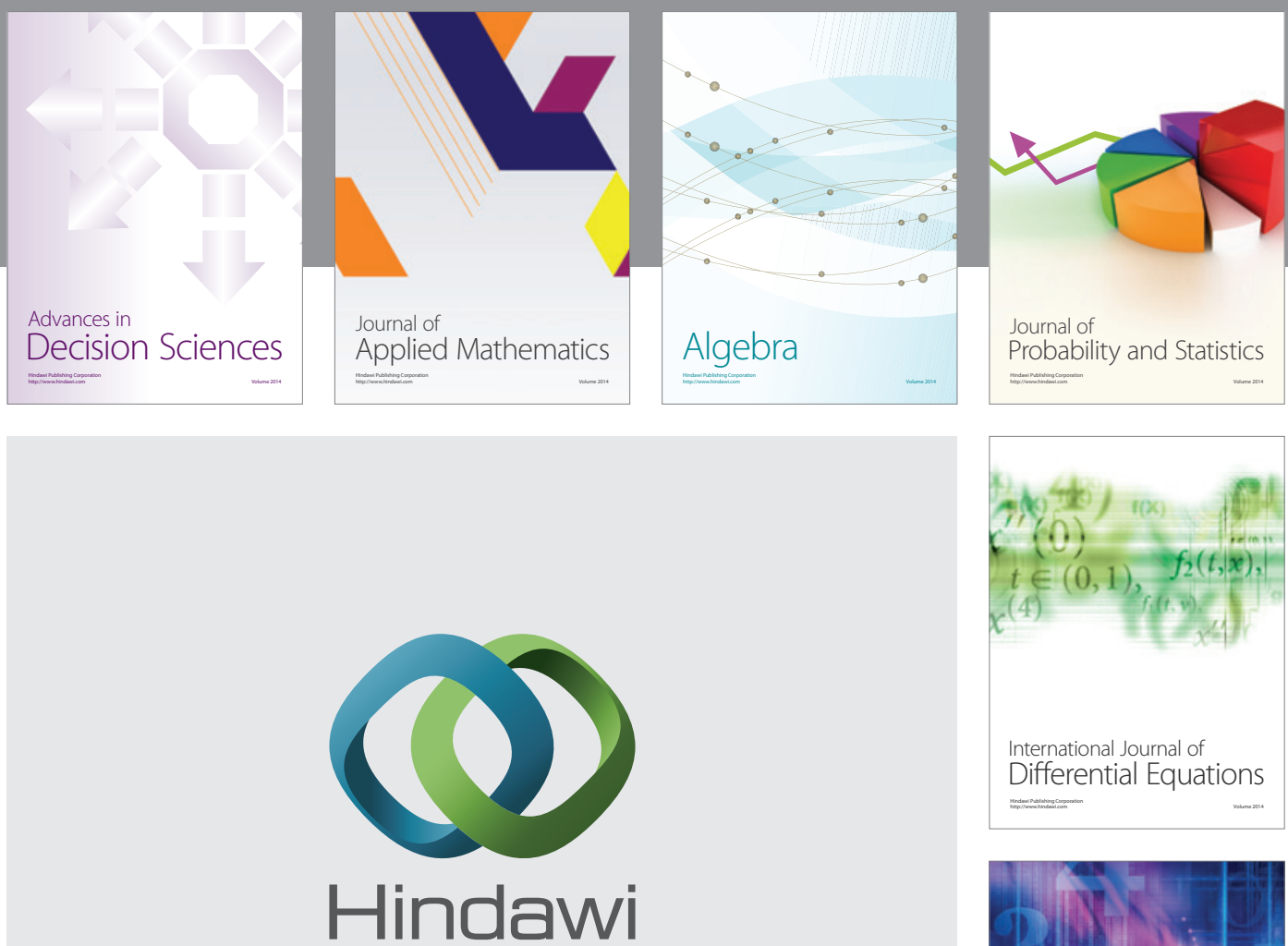

Submit your manuscripts at http://www.hindawi.com
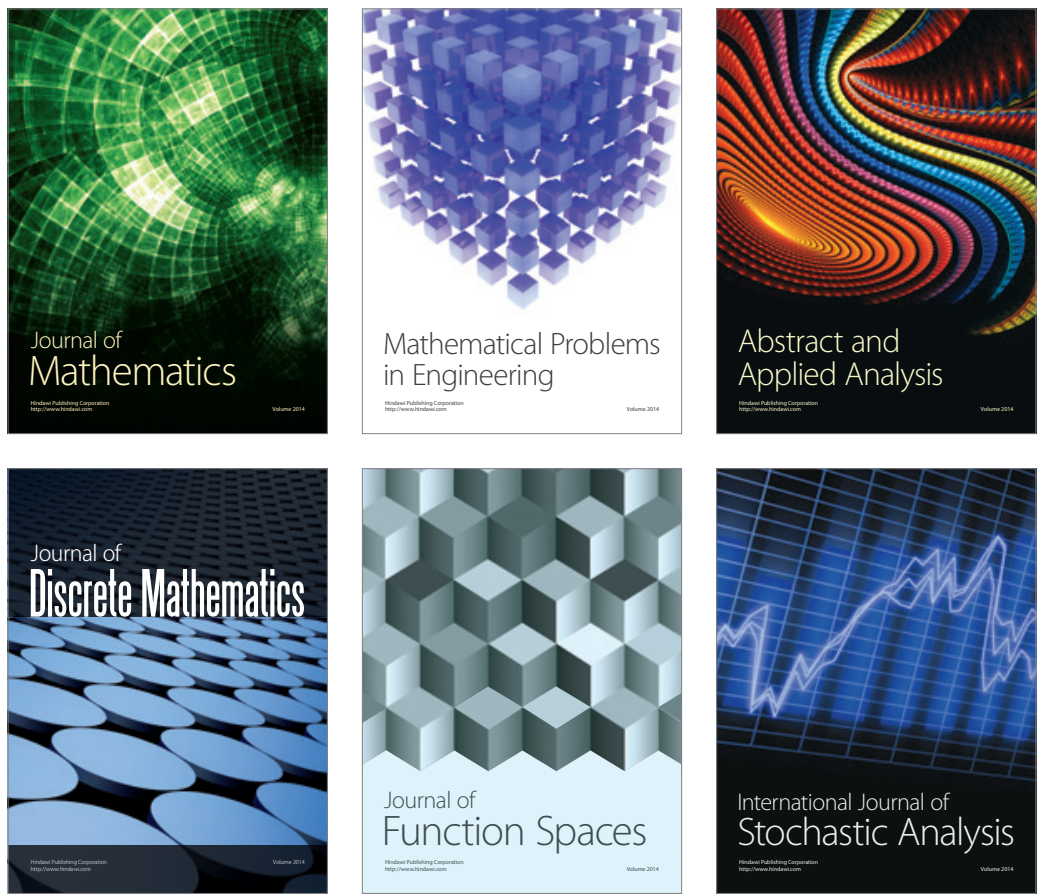

Journal of

Function Spaces

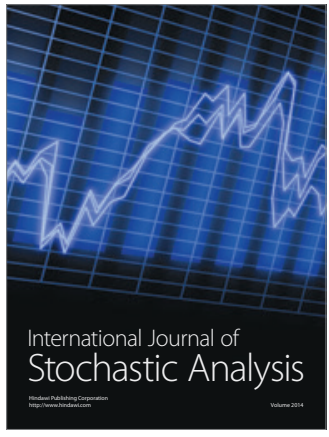

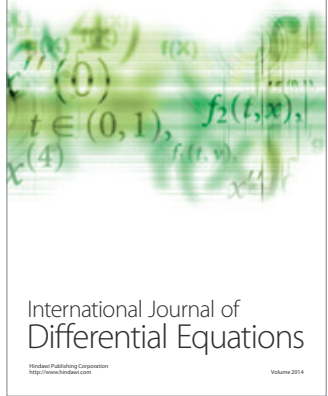
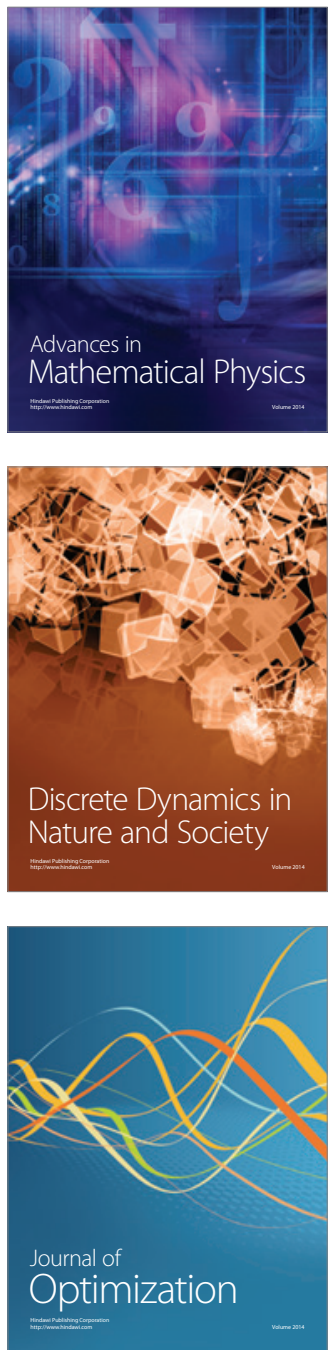